\title{
Anthropometric Indices and Their Relationship With Coronary Artery Diseases
}

\author{
Golnaz Arjmand ${ }^{1}$; Farzad Shidfar ${ }^{2, *}$; Marzieh Molavi Nojoomi ${ }^{3}$; Abdullah Amirfarhangi ${ }^{4}$ \\ ${ }^{1}$ Department of Clinical Nutrition, Tehran University of Medical Sciences, Tehran, IR Iran \\ 2 Department of Nutrition, School of Health, Iran University of Medical Sciences, Tehran, IR Iran \\ 3 Department of Social Medicine, Iran University of Medical Sciences, Tehran, IR Iran \\ Department of Social Medicine, Iran University of Medical Sciences, Tehran, IR Iran
4 Department of Cardiology, Rasoule Akram Hospital, Iran University of Medical Scie \\ ${ }^{*}$ Corresponding author: Farzad Shidfar, Department of Nutrition, Iran University of Medical Sciences, Tehran, IR Iran. Tel: +98-2186704743, Fax: +98-2188622707, \\ E-mail: farzadshidfar@yahoo.com
}

Received: November 3, 2014; Revised: February 8, 2015; Accepted: April 17, 2015

\begin{abstract}
Background: Despite efforts made by the medical community and public health officials for combating 76 obesity, the prevalence of overweight and obesity has increased over the past 20 years. 77 Obesity was associated with some cardiovascular risk factors and increased the incidence of 78 cardiovascular disease. Diagnosis of Coronary Artery Disease (CAD) in patients with obesity 79 will affect secondary prevention of the disease and management programs; for this reason it is 80 necessary to use a suitable method for determining obesity Objectives: The current study aimed to investigate the relationship between some anthropometric indices and Coronary Artery Disease (CAD) risk factors and also to determine predictive factors of CAD among the indices.

Patients and Methods: This cross-sectional study was performed on 300 men and nonpregnant woman with CAD aged 18 - 60 years referred to Hazrate Rasool Hospital in Tehran, Iran. Anthropometric indices, including waist circumference, waist-to-height ratio, Neck Circumference (NC), body mass index and Abdominal Volume Index (AVI) and hematological factors were measured using the standard methods. According to angiography reports, the participants were divided into patients with or without CAD. To evaluate the possible correlations between anthropometric indices and CAD risk factors, Pearson correlation coefficients were used. The Receiver operating characteristic (ROC) curve was used to determine the sensitivity and specificity and the best cut-off point of anthropometric indices.

Results: The mean age of the CAD-positive group was $53.43 \pm 4.96$ years and the mean age of the CAD-negative group was $49.9 \pm 6.83$ years $(\mathrm{P}>0.05)$. All of the anthropometric indices and cardiovascular risk factors were higher in the CAD-positive group, while there was a significance correlation between HDL-c (High-density lipoprotein) and these risk factors $(\mathrm{P}<0.05)$. The area under the curve of AVI had the highest value (0.722) compared to other anthropometric indices. The results of multiple-regression analysis showed that NC was a better predictor of the risk of CAD compared to other anthropometric indices $(\mathrm{P}=0.46, \mathrm{OR}=1.207$ and $\mathrm{CI}=1.004-1.451)$.

Conclusions: According to the results of the study, abdominal obesity is as a risk factor for atherosclerosis. Therefore, the use of a simple, low cost and high precision method for determining visceral obesity and preventing the CAD is warranted.
\end{abstract}

Keywords: Coronary Artery Disease; Abdominal Volume Index, Neck Circumference

\section{Background}

Despite efforts made by the medical community and public health officials for combating obesity, the prevalence of overweight and obesity has increased over the past 20 years (1). Obesity was associated with some cardiovascular risk factors (2) and increased the incidence of cardiovascular disease (3). Diagnosis of Coronary Artery Disease (CAD) in patients with obesity will affect secondary prevention of the disease and management programs; for this reason it is necessary to use a suitable method for determining obesity $(4,5)$. Several methods exist to evaluate weight gain and obesity. Body Mass Index (BMI) is a technique that is widely used to classify weight gain and obesity (6). However, the BMI did not show full measure of body fat $(7,8)$. As a limitation for BMI, normal range for this indicator, which has been introduced by the world health organization is widespread and many who are in this range may increase abdominal fat accumulation and increased risk for the disease (9). Since the central body fat distribution is more atherogenic properties than peripheral obesity, much attention has been focused on methods to measure central obesity. Fat deposits in the abdomen and central body releases free fatty acids into the peripheral blood flow and portal blood which can lead to insulin resistance in liver and muscle cells and increase release of triglyceride-rich lipoproteins and very low density from the liver and thus produce dense low-density lipoproteins $(10,11)$. Therefore, the Waist Circumference (WC) and Waist-to-Height Ratio (WHtR) are clinical tools that can be used for diagnosis of abdominal obesity (12). Although the majority of studies determine 
that the WC is a better indicator of obesity than BMI (1316), this finding in Asian (17) studies and Pacific (18) is not approved and obesity indices that can predict the risk of cardiovascular disease remain as a controversial issue (19). Another indicator used in the evaluation of abdominal obesity is abdominal volume index that is defined by Guerrero-Romero and Rodriguez-Moran (20).

Although obesity and ectopic fat accumulation in the central of body are associated with many of the risk factors of coronary heart disease (21), studies have shown that increased upper body fat distribution increases the risk of cardiovascular disease, and Neck Circumference (NC) is considered as an indicator to measure these risk factors $(22,23)$. The report indicates that the release of free fatty acids from the subcutaneous fat of the upper body is higher than that in the lower body (24). Growing evidences from population studies hypothesize that people who have a greater NC are at the higher risk of heart disease (25). Results from the Framingham Heart Study showed that NC rather than BMI index was independently associated with the risk of cardiovascular disease (26). However, the role of NC in predicting cardiovascular risk factors is not well-understood.

Since the anthropometric indices are seem easier, cheaper and more readily available than ultrasound techniques and limited studies were examined the relationship between obesity and atherosclerosis especially in Iran; the aim of this study was to investigate the relationship between some anthropometric indices and CAD risk factors and also to determine predictive factors among them.

\section{Objectives}

The current study aimed to investigate the relationship between some anthropometric indices and Coronary Artery Disease (CAD) risk factors and also to determine predictive factors of CAD among the indices.

\section{Patients and Methods}

This cross-sectional study was performed on 300 patients with CAD referred to Hazrate Rasool Hospital in Tehran City, Iran during a 6-month period from July to December 2012. All patients with the inclusion criteria due to suspected ischemia underwent coronary angiography were enrolled in the study. Inclusion criteria included accepting contributions, history of chronic stable angina, availability of previous medical history and physical examination.

Exclusion criteria included candidates for coronary angiography for reasons unrelated to CAD, patients with chronic heart failure, myocardial infarction or unstable angina and people who participate in weight loss programs or related drugs for weight loss and lipid lowering drugs. From a total of patients referred to angiography, 350 patients were invited to participate in the study, of which 300 accepted the invitation and were enrolled in the study. The age range is between 18 - 60 years. At the first meeting with the patients, information on the application and angiography, previous medical history and medications (such as weight loss drugs) were collected.

Both systolic and diastolic blood pressure levels were measured using a gauge of the right arm and after $15 \mathrm{~min}$ utes rest with a mercury sphygmomanometer in a sitting position. Systolic blood pressure level less than $130 \mathrm{~mm}$ $\mathrm{Hg}$ and diastolic blood pressure level less than $85 \mathrm{~mm} \mathrm{Hg}$ were considered as normal blood pressure.

\subsection{Anthropometric Indices}

Weight was measured by using a digital scale (Hanson, Watford, Hertforshire, England), with precision of $100 \mathrm{~g}$ and a coefficient of variation $>1 \%$, without shoes with a thin coating. The standing height was measured as the four points of the body (heel, buttock, back and head) were in contact with the meter gauge, shoulders was relax, heels together and head was in the direction of Frankfurt. Body mass index was calculated by dividing weight in kilograms by square of height in meters. Waist circumference was measured horizontally halfway between the lower border of the rib cage and the iliac crest using a rubber measuring tape. Neck circumference was measured using the calibrated tape perpendicular to the axis of the neck just below the laryngeal prominence (27).

Abdominal Volume Index (AVI) was calculated from the following equation:

1)

$$
\mathrm{AVI}=\left[2 \mathrm{WC}^{2}+0.7(\mathrm{WC}-\mathrm{HC})^{2}\right] \times 1000
$$

Fat mass was determined by Bioelectrical Impedance Analysis (BIA).

\subsection{Blood Measurements}

Blood samples were collected after overnight fasting. Triglycerides and lipoprotein levels were measured. Total Cholesterol (TC), Fasting Blood Sugar (FBS), Triglyceride (TG), HDL-C (High-density lipoprotein), LDL-C (Low density lipoprotein) and BUN (Blood Urea Nitrogen) and Creatinine ( $\mathrm{Cr}$ ) levels were determined by enzymatic procedures (Pars Azmon Kit, Tehran, Iran).

\subsection{Coronary Angiography}

Electrocardiogram (ECG) changes suggestive of CAD was defined as SD depression $>0.5 \mathrm{~mm}$ or T inversion $>$ $2 \mathrm{~mm}$. The Judkins technique of angiography was performed through the femoral artery and at least four views of the left coronary artery and two views of the right coronary artery were obtained; CAD was defined as stenosis of more than $50 \%$ of the vessel lumen diameter.

\subsection{Statistical Analyses}

Statistical analyses were performed using SPSS software version 18.0 (Chicago, Illinois, USA). An unpaired Student t-test was used to compare the continuous 
variables. To evaluate the possible correlations between anthropometric indices and CVD risk factors, Pearson correlation coefficients were used. The ROC curve was used to determine the sensitivity and specificity and the best cut-off point of anthropometric indices. This test was used as a backup system in case that the target was determined the best diagnostic criteria. The best cut-off point of the test parameters was derived based on the Youden index. $\mathrm{P}<0.05$ is defined as the level of significance for all statistical analyses.

\section{Results}

Three hundred patients (155 men and 145 women) with stable angina who underwent angiography (age 18 - 60 years) were enrolled in the study. Of these participants, 231 and 68 patients were in the CAD-positive and -negative groups, respectively. The mean age of the CAD-positive group was $53.43 \pm 4.96$ years and -the mean age of the CAD-negative group was $49.9 \pm 6.83$ years $(\mathrm{P}>0.05)$. Characteristics of the study population are presented in Table 1. The patient with CAD had significantly higher levels of systolic blood pressure $(\mathrm{P}=0.000)$, fasting blood glucose $(\mathrm{P}=0.004)$ and LDL-c $(\mathrm{P}=0.000)$.

Table 2 shows the correlation among the variables between the two groups. As shown in Table 2, BMI, WC, WtHR, PBF (Percent of body fat) and NC were positively correlated with SBP. In patient without CAD only PBF was not correlated with SBP. In patient with CAD, BMI was positively correlated with SBP, DBP, LDL-c, TC.HDLc, LDL-c/HDL-c and INR (international normalized ratio). In the CAD group WC was positively correlated with SBP, DBP, TC/HDL-c and LDL-c/HDL-c and negatively correlated with HDL-c. Waist to hip ratio in the CADpositive group was positively correlated with SBP, TG/ HDL-c, TC/HDL-c, LDL-c, and HDL-c. An inverse correlation was found between WtHR and HDL-c. In the CADpositive group, PBF was positively correlated with SBP, FBS, Tchol, TG, TG/HDL-c, TC/HDL-c, LDL-c, HDL-c, INR and PT (Prothrombin time). In the CAD-positive group, NC indices were positively correlated with SBP, DBP, Tchol, TG, LDL-c, INR and PT and negatively correlated with TG and HDL-c.

Table 3 shows that the area under the curve of AVI has the highest value compared to other anthropometric indices, while the lowest value belongs to waist to hip ratio. As shown in Table 3, the waist to height ratio and NC were the most sensitive indicators while waist-to-hip ratio had the lowest sensitivity.

The results of multiple regression analysis showed that $\mathrm{NC}$ is a better predictor of the risk of CAD compared to other anthropometric indices $(\mathrm{P}=0.46, \mathrm{OR}=1.207$ and $\mathrm{CI}=1.004-1.451$ )

Table 1. Characteristics of the Study Population ${ }^{a}$

\begin{tabular}{|c|c|c|c|}
\hline Variables & CAD Positive & CAD Negative & P Value \\
\hline WC, $\mathbf{c m}$ & $98.69 \pm 9.19$ & $91.99 \pm 10.31$ & 0.106 \\
\hline WtHR & $0.59 \pm 0.066$ & $0.56 \pm 0.057$ & 0.534 \\
\hline PBF, \% & $33.37 \pm 7.38$ & $31.13 \pm 6.74$ & 0.166 \\
\hline BMI, $\mathrm{kg} / \mathrm{m}^{2}$ & $30.76 \pm 4.63$ & $28.13 \pm 4.88$ & 0.805 \\
\hline $\mathrm{NC}, \mathrm{cm}$ & $39.68 \pm 2.61$ & $36.36 \pm 3.07$ & 0.010 \\
\hline $\mathrm{AVI}, \mathrm{cm}$ & $19.86 \pm 3.59$ & $17.32 \pm 4.08$ & 0.133 \\
\hline SBP, mmhg & $149.92 \pm 21.47$ & $135.79 \pm 16.26$ & 0.000 \\
\hline DBP, mmhg & $91.08 \pm 56.12$ & $79.39 \pm 14.27$ & 0.390 \\
\hline FBS, $\mathrm{mmol} / \mathrm{L}$ & $139.73 \pm 56.03$ & $113.47 \pm 50.77$ & 0.004 \\
\hline $\mathrm{TC}, \mathrm{mmol} / \mathrm{L}$ & $180.63 \pm 37.45$ & $158.89 \pm 34.72$ & 0.145 \\
\hline TG, mmol/L & $180.68 \pm 75.42$ & $159.70 \pm 77.53$ & 0.705 \\
\hline LDL-c, mmol/L & $111.39 \pm 26.74$ & $91.17 \pm 15.31$ & 0.000 \\
\hline HDL-c, mmol/L & $34.67 \pm 8.79$ & $36.66 \pm 10.30$ & 0.356 \\
\hline BUN, mmol/L & $15.81 \pm 4.26$ & $13.16 \pm 3.93$ & 0.748 \\
\hline $\mathrm{Cr}, \mathrm{mmol} / \mathrm{L}$ & $1.16 \pm 0.26$ & $0.95 \pm 0.22$ & 0.528 \\
\hline
\end{tabular}

\footnotetext{
a Abbreviations: AVI, Abdominal volume index; BUN, Blood urea nitrogen; BMI, Body mass index; CAD, coronary artery disease; Cr, Creatinine; DBP, Diastolic blood pressure; FBS, Fasting blood sugar; HDL-c, High density lipoprotein; LDL-c, Low density lipoprotein; NC, Neck circumferences; PBF, Percent of body fat; SBP, Systolic blood pressure; TC, Total cholesterol; TG, triglyceride; WC, Waist circumferences; WtHR, Waist to hip ratio.
} 
Arjmand Get al.

\begin{tabular}{|c|c|c|c|c|c|c|c|c|c|c|c|c|c|}
\hline \multirow[t]{2}{*}{ Variables } & & \multicolumn{2}{|c|}{ BMI } & \multicolumn{2}{|c|}{ WC } & \multicolumn{2}{|c|}{ WtHR } & \multicolumn{2}{|c|}{ PBF } & \multicolumn{2}{|c|}{ NC } & \multicolumn{2}{|c|}{ AVI } \\
\hline & & $\mathbf{P}^{\mathrm{b}}$ & $\mathbf{N}^{\mathrm{C}}$ & $\mathbf{P}$ & $\mathbf{N}$ & $\mathbf{P}$ & $\mathbf{N}$ & $\mathbf{P}$ & $\mathbf{N}$ & $\mathbf{P}$ & $\mathbf{N}$ & $\mathbf{P}$ & $\mathbf{N}$ \\
\hline \multirow[t]{2}{*}{ SBP } & $\mathbf{R}^{\mathrm{d}}$ & 0.385 & 0.352 & 0.260 & 0.345 & 0.184 & 0.355 & 0.234 & 0.055 & 0.290 & 0.360 & 0.251 & 0.317 \\
\hline & $\mathbf{P}^{\mathrm{e}}$ & 0.000 & 0.003 & 0.000 & 0.004 & 0.005 & 0.355 & 0.000 & 0.657 & 0.000 & 0.003 & 0.000 & 0.009 \\
\hline \multirow[t]{2}{*}{ DBP } & $\mathbf{r}$ & 0.538 & 0.000 & 0.186 & 0.453 & 0.090 & 0.457 & 0.077 & 0.032 & 0.291 & 0.429 & 0.167 & 0.446 \\
\hline & $\mathbf{P}$ & 0.000 & 0.001 & 0.005 & 0.000 & 0.173 & 0.000 & 0.244 & 0.793 & 0.000 & 0.000 & 0.011 & 0.000 \\
\hline \multirow[t]{2}{*}{ FBG } & $\mathbf{r}$ & 0.096 & -0.054 & 0.036 & -0.153 & 0.074 & -0.165 & 0.181 & -0.30 & -0.021 & 0.114 & 0.034 & -0.140 \\
\hline & $\mathbf{P}$ & 0.145 & 0.661 & 0.590 & 0.213 & 0.263 & 0.179 & 0.006 & 0.807 & 0.751 & 0.353 & 0.606 & 0.256 \\
\hline \multirow[t]{2}{*}{ TC } & $\mathbf{r}$ & 0.107 & 0.123 & 0.037 & 0.051 & 0.004 & 0.024 & 0.203 & 0.090 & 0.156 & 0.145 & 0.046 & -0.049 \\
\hline & $\mathbf{P}$ & 0.105 & 0.318 & 0.571 & 0.680 & 0.953 & 0.843 & 0.002 & 0.464 & 0.018 & 0.238 & 0.488 & 0.690 \\
\hline \multirow[t]{2}{*}{ TG } & $\mathbf{r}$ & 0.038 & 0.502 & 0.024 & 0.446 & 0.052 & 0.385 & 0.251 & 0.186 & -0.100 & 0.525 & 0.033 & 0.434 \\
\hline & $\mathbf{P}$ & 0.566 & 0.000 & 0.720 & 0.000 & 0.433 & 0.001 & 0.000 & 0.129 & 0.131 & 0.000 & 0.621 & 0.000 \\
\hline \multirow[t]{2}{*}{ LDL-c } & $\mathbf{r}$ & 0.167 & 0.201 & 0.079 & 0.178 & -0.012 & 0.216 & 0.122 & 0.293 & 0.217 & 0.256 & 0.080 & 0.210 \\
\hline & $\mathbf{P}$ & 0.011 & 0.100 & 0.232 & 0.147 & 0.854 & 0.077 & 0.65 & 0.015 & 0.001 & 0.035 & 0.228 & 0.086 \\
\hline \multirow[t]{2}{*}{ HDL-c } & $\mathbf{r}$ & -0.112 & -0.298 & -0.224 & -0.378 & -0.224 & -0.236 & -0.121 & -0.142 & 0.058 & -0.470 & -0.223 & -0.382 \\
\hline & $\mathbf{P}$ & 0.091 & 0.014 & 0.001 & 0.001 & 0.001 & 0.053 & 0.066 & 0.247 & 0.380 & 0.000 & 0.001 & 0.001 \\
\hline \multirow[t]{2}{*}{ TG/HDL-c } & $\mathbf{r}$ & 0.092 & 0.610 & 0.115 & 0.460 & 0.161 & 0.460 & 0.280 & 0.245 & -0.148 & 0.641 & 0.123 & 0.574 \\
\hline & $\mathbf{P}$ & 0.161 & 0.000 & 0.080 & 0.000 & 0.014 & 0.000 & 0.000 & 0.044 & 0.025 & 0.000 & 0.063 & 0.000 \\
\hline \multirow[t]{2}{*}{ TC/HDL-c } & $\mathbf{r}$ & 0.169 & 0.433 & 0.208 & 0.350 & 0.198 & 0.281 & 0.290 & 0.238 & 0.040 & 0.535 & 0.214 & 0.359 \\
\hline & $\mathbf{P}$ & 0.010 & 0.000 & 0.001 & 0.003 & 0.003 & 0.020 & 0.000 & 0.051 & 0.547 & 0.000 & 0.001 & 0.003 \\
\hline \multirow[t]{2}{*}{ LDL-c/HDL-c } & $\mathbf{r}$ & 0.213 & 0.535 & 0.211 & 0.599 & 0.154 & 0.475 & 0.199 & 0.385 & 0.085 & 0.196 & 0.212 & 0.635 \\
\hline & $\mathbf{P}$ & 0.001 & 0.000 & 0.001 & 0.000 & 0.019 & 0.000 & 0.002 & 0.001 & 0.196 & 0.000 & 0.001 & 0.000 \\
\hline \multirow[t]{2}{*}{ BUN/Cr } & $\mathbf{r}$ & 0.101 & 0.007 & -0.001 & -0.142 & 0.247 & -0.037 & 0.081 & 0.185 & 0.045 & -0.87 & 0.001 & -0.151 \\
\hline & $\mathbf{P}$ & 0.125 & 0.956 & 0.994 & 0.247 & 0.627 & 0.756 & 0.221 & 0.130 & 0.499 & 0.480 & 0.984 & 0.218 \\
\hline
\end{tabular}

Table 3. The Cut-Off Point to Maximize Sensitivity and Specificity and Area Under the Curve for Each Anthropometric Index in the CAD-Positive Group ${ }^{\text {a }}$

\begin{tabular}{lccccccc}
\hline Anthropometric Indices & AUC & Cut-Off Point & Sensitivity & 1-Specifity & Upper-Bound & Lower-Bound & P \\
\hline WC & 0.720 & 95.75 & 0.693 & 0.368 & 0.794 & 0.647 & 0.000 \\
Waist to Height Ratio & 0.720 & 0.575 & 0.706 & 0.324 & 0.769 & 0.618 & 0.000 \\
BMI & 0.681 & 28.87 & 0.662 & 0.338 & 0.785 & 0.604 & 0.000 \\
NC & 0.711 & 38.25 & 0.706 & 0.338 & 0.789 & 0.634 & 0.000 \\
Percent of Body Fat & 0.597 & 30.35 & 0.615 & 0.426 & 0.672 & 0.523 & 0.015 \\
AVI & 0.722 & 18.64 & 0.697 & 0.324 & 0.797 & 0.646 & 0.000 \\
\hline
\end{tabular}

a Abbreviations: AUC, Area under curve; AVI, Abdominal volume index; BMI, Body mass index; WC, Waist circumferences.

\section{Discussion}

The increasing prevalence of obesity and related chronic diseases such as diabetes, cardiovascular disease, cancer and mortality make it continues to be at the forefront of health problems and plan for the prevention and treatment of obesity will be a major health priority. The first step in planning for health is screening and identifica- 
Arjmand Get al.

tion of obesity with an easy and accurate manner. Therefore, the correlation between anthropometric indices and cardiovascular disease risk factors has been examined to determine the best factor.

In brief, the results of this study showed that the mean age \pm SD of the CAD-positive group was $53.43 \pm 4.96$ and the mean age for the CAD-negative group was $49.9 \pm 6.83$ years $(\mathrm{P}>0.05)$. All of the anthropometric indices and cardiovascular risk factors in the CAD-positive group were higher compared to the CAD-negative one, while there was a significance correlation between HDL-c and these risk factors $(\mathrm{P}<0.05)$. The area under the curve of AVI was the highest value (0.722) compared to other anthropometric indices. The results of multiple regression analysis showed that NC was a better predictor of CAD compared to other anthropometric indices $(\mathrm{P}=0.46$, OR $=1.207$ and $\mathrm{CI}=1.004-1.451$ ).

A number of studies have suggested that WC is a good indicator for predicting the risk of cardiovascular disease $(28,29)$, while other studies have shown that waisthip ratio is a better indicator for predicting the risk of cardiovascular disease compared to WC and BMI (30). Yusuf and colleagues reported in their study that, waist-toheight ratio as an indicator of central obesity is a better predictor of cardiovascular events compared to WC and BMI in both sexes (31). In our study it was shown that WC was positively associated with the risk of cardiovascular disease, but waist-to-height index as a simple indicator to measure, has a stronger predictive power (due to a higher area under the curve). The findings of this study were strengthened with the studies that have reported an association between BMI and anthropometric indices. Consistent with the present study Moy and colleagues compared two indices of WC and waist-to-hip ratio with BMI in 20 to 58 years Malaysian men and women, and concluded that WC is superior in obesity screening than waist-hip ratio and can be used to replace BMI in weight management (32). In our study, the cut-off value of WC for predicting risk of coronary artery disease was 75.95 $\mathrm{cm}$. The only prospective cohort study that has been conducted is cited WC index cut-off value for predicting cardiovascular risk equal to $50.94 \mathrm{~cm}$ (33). The interheart study showed that waist-hip index is the strongest predictor of myocardial infarction in both sexes. In this study, the preference of waist-to-hip ratio index to WC alone is associated to hip circumference, because higher hip circumference was significantly increased the risk of cardiovascular disease (15). Contradictory conclusions about the risk of hip and cardiovascular disease are about that analysis of cross-sectional data cannot be associated with an estimated incidence of cardiovascular disease (34). To have a good choice to determine the best cut-off point for anthropometric measures, some factors such as gender, age, ethnicity, and the prevalence of risk factors should be considered. In women, younger adults and in people with more common risk factors such as lipid disorder, the most appropriate cut-off points is at lower levels; while in older people and having a less common risk factors such as number of risk factors (as a surrogate for risk of cardiovascular disease) the higher cut-off point is more appropriate $(35,36)$. Cut-off points for anthropometric indices have been obtained from a limited number of cross-sectional analyses, while this power set is much stronger in cohort studies. On other hand, the reason for the difference in cut-off points is the difference between general and central obesity in diverse population (37). In this study, we used ROC analysis to determine the cut-off point, which is how dependent is anthropometric distribution of the samples. This means that with the increase in population distribution rate, the cut-off points derived from ROC analysis were also raised (38). In our study, the cut-off value of WC to predict the risk of CAD was determined $95.75 \mathrm{~cm}$. The only prospective cohort study that has been conducted in Iran is cited the cut-off values of WC for predicting cardiovascular risk equal to $94.5 \mathrm{~cm}$ (33). We suggest the cut-off value for waist-to-height index for the Iranian population equal to 0.57 . This research listed the amount of the waist-to-height ratio equal to 0.62 (33). In this study, the higher WC index in both genders can be associated with a higher prevalence of general and abdominal obesity in Iranian population. The most recent national assessment of Iranian adult population showed that the mean values for WC for men and women were $86.6 \mathrm{~cm}$ and $89.6 \mathrm{~cm}$, respectively, which can be influenced by genetic factors, low literacy rates, the above fertility rates and sedentary lifestyle (39). A number of mechanisms available to support the findings of our study are as follows: unlike BMI, WC index shows abdominal fat distribution in the area that is associated with the risk of cardiovascular disease more than the body weight (40). Body mass index is unable to detect individuals with excess fat and high muscle mass, with this assumption; based on their BMI risk of cardiovascular disease is similar if both are the same person's weight and height (41). However, the WC index, is a simple indicator to measure abdominal obesity; however, it seems that people with similar WC has also similar risk of cardiovascular disease (regardless of the difference in height) (42). On the other hand, risk of diabetes and high blood pressure in individuals with short stature is more than tall people (43). Thus, studies and analysis conducted has shown that the predictive power of WC for risk of cardiovascular disease is enhanced when it corrected by height and hip circumference (20). Finally, there is the fact that with the change in body size index, waist-hip ratio remains constant, because both waist and hip parameters are changed proportion to each other. However, any small change in the WC will cause to change waist-toheight ratio, because adult height is fixed.

Studies show that, BMI along with increased WC can predict the incidence of cardiovascular disease than any single indicator alone (27). Our study has shown that in the CAD-positive group AVI was negatively correlated with the HDL-c parameter. While this correlation be- 
tween systolic and diastolic blood pressure and the ratio of TC/ HDL-c and LDL-c/HDL-c was significantly positive. Also, the results of our study showed that there was a significant positive association between WC, hip circumference, waist-to-hip ratio, waist to height ratio, body fat percentage and BMI and the CAD. According to the results of our study, AVI has the highest area under the curve among other anthropometric indices (0.772), the highest specificity (69\%) and the lowest sensitivity (32\%).

In a study done in 2003 that was conducted on 746 men and nonpregnant women with CAD, the best cut-off point for AVI index to determine overweight and obesity is equal to 5.24 liters and this index has a significant positive relationship with glucose intolerance and type 2 diabetes (20). Studies on this indicator are very limited.

The results of our study showed that the NC has a positive and significant relationship with LDL-c and a negative relationship with TG/HDL ratio. Also, our study showed that in the CAD-positive group, NC has a strong positive and significant association with other anthropometric indices including WC, hip circumference, waistto-hip ratio, waist-to-height ratio and the percentage of body fat. These findings are consistent with the findings of other studies.

The femoral and NC is considered as an indicator of subcutaneous fat distribution in upper and lower of body. Central obesity, especially high levels of visceral fat in the upper body is accompanied by bad metabolic complications such as insulin resistance, diabetes, high blood pressure and an increase in blood TG levels, while these effects are low in lower body obesity. Hence, NC as a representative of the accumulation of fat in the upper body is independently associated with the risk of cardiovascular disease (20).

Preis and colleagues reported in 2010 that the NC, as an indicator of upper body fat distribution below the skin, is directly associated with cardiovascular risk factors. Ben-Noun and Laor reported in their study that NC is associated with high blood pressure and other metabolic syndrome risk factors, including cholesterol-LDL, cholesterol-HDL, TG, and TC. This is a cohort study of 364 participants. The results of their study showed that NC change over time was significantly in line with changes in BMI, WC, waist-to-hip ratio, TC, cholesterol-LDL, TG, glucose and uric acid but was not correlated with changes in cholesterol-HDL (44).

Wang and colleagues showed in their study that, NC as a place to store fat in the upper body, was positively associated with visceral fat in the abdomen in Chinese adult population (45). Waist circumference as an index of anthropometric indices easily can be affected by factors such as satiety or full stomach, respiratory activity and wearing thick clothing; confounding the effect of these factors can be prevented by replacing NC instead of WC. Thus, it can be concluded that the NC can be considered as a valid and more accurate indicator of central obesity (44).

According to the results of ROC curve in our study, NC has a greater area under the curve than waist-to-height, waist-to-hip ratio and BMI. This is also consistent with our study, Zhu and colleagues showed that, NC has a greater area under the curve than waist-to-height, waist-to-hip ratio and BMI (43).

Among the existing mechanisms of the association between NC and risk of cardiovascular disease can be cited to impaired respiratory diseases in people with greater NC, because interrupted breathing during sleep or sleep apnea is associated with an increase in blood pressure, cholesterol levels, reducing cholesterol-LDL, diabetes and insulin resistance $(47,48)$. On the other hand the increase in the accumulation of fat in the upper body is accompanied by the increased levels of free fatty acids in plasma; the increase in free fatty acids was associated with increased oxidative stress and damage to the vessel wall, both of which increase the risk of high blood pressure. Since obese people have fat stored between 2 to 3 times higher than normal; thus, the levels of circulating free fatty acids in them are the most. These fats are delivered to the liver and increase the concentration of VLDL (Very Low Density Lipoprotein) and LDL cholesterol produced by the liver $(49,50)$.

Therefore, and due to the mechanisms mentioned above, it can be expressed that NC is linked to cardiovascular disease risk factors.

The principal limitation of this study was the relatively short duration of follow-up. Lack of data on insulin values might be another important limitation of this study, as differences in the effects of BMI and waist might be mediated by insulin resistance.

According to the results of the study, abdominal obesity is as a risk factor for atherosclerosis. Therefore, the use of a simple, low cost and high precision method for determining visceral obesity and preventing the CAD is warranted.

\section{Acknowledgements}

The authors would like to thank all personnel who working at angiography section of Hazrate Rasool Hospital. This research was supported by Tehran University of Medical Sciences.

\section{Authors' Contributions}

Farzad Shidfar: Critical revision of the manuscript for important intellectual content, study supervision, administrative, technical, and material support. Golnaz Arjmand: Study concept and design, acquisition of data, drafting of the manuscript. Marzieh Molavi Nojoomi: Statistical analysis.Abdolahh Amir Farhangi: Critical revision of the manuscript for important intellectual content.

\section{References}

1. Ogden CL, Flegal KM, Carroll MD, Johnson CL. Prevalence and 
trends in overweight among US children and adolescents, 19992000. JAMA. 2002;288(14):1728-32.

2. Grundy SM. Obesity, metabolic syndrome, and coronary atherosclerosis. Circulation. 2002;105(23):2696-8.

3. Kannel WB, Gordon T, Castelli WP. Obesity, lipids, and glucose intolerance. The Framingham Study. Am J Clin Nutr. 1979;32(6):1238-45.

4. World Health Organization.. Physical status: The use of and interpretation of anthropometry, Report of a WHO Expert Committee.; 1995.

5. Wood D, De Backer G, Faergeman O, Graham I, Mancia G, Pyorala K. Prevention of coronary heart disease in clinical practice. Summary of recommendations of the Second Joint Task Force of European and other Societies on Coronary Prevention.J Hypertens. 1998;16(10):1407-14.

6. Eknoyan G. Adolphe Quetelet (1796-1874)--the average man and indices of obesity. Nephrol Dial Transplant. 2008;23(1):47-51.

7. Gallagher D, Visser M, Sepulveda D, Pierson RN, Harris T, Heymsfield SB. How useful is body mass index for comparison of body fatness across age, sex, and ethnic groups? Am J Epidemiol. 1996;143(3):228-39.

8. Jackson AS, Stanforth PR, Gagnon J, Rankinen T, Leon AS, Rao DC, et al. The effect of sex, age and race on estimating percentage body fat from body mass index: The Heritage Family Study. Int $J$ Obes Relat Metab Disord. 2002;26(6):789-96.

9. Hsieh SD, Yoshinaga H, Muto T. Waist-to-height ratio, a simple and practical index for assessing central fat distribution and metabolic risk in Japanese men and women. Int $J$ Obes Relat Metab Disord. 2003;27(5):610-6.

10. Vinik AI. The metabolic basis of atherogenic dyslipidemia. Clin Cornerstone. 2005;7(2):27-35.

11. Kang HS, Gutin B, Barbeau P, Litaker MS, Allison J, Le NA. Low-density lipoprotein particle size, central obesity, cardiovascular fitness, and insulin resistance syndrome markers in obese youths. Int J Obes Relat Metab Disord. 2002;26(8):1030-5.

12. Ho SY, Lam TH, Janus ED. Waist to stature ratio is more strongly associated with cardiovascular risk factors than other simple anthropometric indices. Ann Epidemiol. 2003;13(10):683-91.

13. Pouliot MC, Despres JP, Lemieux S, Moorjani S, Bouchard C, Tremblay A, et al. Waist circumference and abdominal sagittal diameter: best simple anthropometric indexes of abdominal visceral adipose tissue accumulation and related cardiovascular risk in men and women. Am J Cardiol. 1994;73(7):460-8.

14. Ledoux M, Lambert J, Reeder BA, Despres JP. A comparative analysis of weight to height and waist to hip circumference indices as indicators of the presence of cardiovascular disease risk factors. Canadian Heart Health Surveys Research Group. CMAJ. 1997;157 Suppl 1:S32-8.

15. Dobbelsteyn CJ, Joffres MR, MacLean DR, Flowerdew G. A comparative evaluation of waist circumference, waist-to-hip ratio and body mass index as indicators of cardiovascular risk factors. The Canadian Heart Health Surveys. Int J Obes Relat Metab Disord. 2001;25(5):652-61.

16. Seidell JC, Cigolini M, Charzewska J, Ellsinger BM, di Biase G. Fat distribution in European women: a comparison of anthropometric measurements in relation to cardiovascular risk factors. Int J Epidemiol. 1990;19(2):303-8.

17. Lin WY, Lee LT, Chen CY, Lo H, Hsia HH, Liu IL, et al. Optimal cut-off values for obesity: using simple anthropometric indices to predict cardiovascular risk factors in Taiwan. Int J Obes Relat Metab Disord. 2002;26(9):1232-8.

18. Welborn TA, Dhaliwal SS, Bennett SA. Waist-hip ratio is the dominant risk factor predicting cardiovascular death in Australia. Med J Aust. 2003;179(11-12):580-5.

19. Singh A, Singh SP, Kaur A. Assessment of Health Status by BMI and BP among Adult Men from Punjab, India. J Life Sci. 2011;3(1):65-7.

20. Guerrero-Romero F, Rodriguez-Moran M. Abdominal volume index. An anthropometry-based index for estimation of obesity is strongly related to impaired glucose tolerance and type 2 diabetes mellitus. Arch Med Res. 2003;34(5):428-32.

21. Ferrannini E, Sironi AM, Iozzo P, Gastaldelli A. Intra-abdominal adiposity, abdominal obesity, and cardiometabolic risk. Eur Heart J Suppl. 2008;10(suppl B):B4-B10.

22. Sjostrom CD, Hakangard AC, Lissner L, Sjostrom L. Body compart- ment and subcutaneous adipose tissue distribution-risk factor patterns in obese subjects. Obes Res. 1995;3(1):9-22.

23. Sjostrom CD, Lissner L, Sjostrom L. Relationships between changes in body composition and changes in cardiovascular risk factors: the SOS Intervention Study. Swedish Obese Subjects. Obes Res. 1997;5(6):519-30.

24. Jensen MD. Lipolysis: contribution from regional fat. Annu Rev Nutr. 1997;17:127-39.

25. Kurtoglu S, Hatipoglu N, Mazicioglu MM, Kondolot M. Neck circumference as a novel parameter to determine metabolic risk factors in obese children. Eur J Clin Invest. 2012;42(6):623-30.

26. Preis SR, Pencina MJ, D'Agostino RS, Meigs JB, Vasan RS, Fox CS Neck circumference and the development of cardiovascular disease risk factors in the Framingham Heart Study. Diabetes Care. 2013;36(1):e3.

27. Ben-Noun LL, Laor A. Relationship between changes in neck circumference and cardiovascular risk factors. Exp Clin Cardiol. 2006;11(1):14-20.

28. Gelber RP, Gaziano JM, Orav EJ, Manson JE, Buring JE, Kurth T. Measures of obesity and cardiovascular risk among men and women. J Am Coll Cardiol. 2008;52(8):605-15.

29. Moy FM, Atiya AS. Waist circumference as a screening tool for weight management: evaluation using receiver operating characteristic curves for Malay subjects. Asia Pac J Public Health. 2003;15(2):99-104.

30. Hadaegh F, Zabetian A, Sarbakhsh P, Khalili D, James WP, Azizi F. Appropriate cutoff values of anthropometric variables to predict cardiovascular outcomes: 7.6 years follow-up in an Iranian population. Int J Obes (Lond). 2009;33(12):1437-45.

31. Yusuf S, Hawken S, Ounpuu S, Bautista L, Franzosi MG, Commerford $\mathrm{P}$, et al. Obesity and the risk of myocardial infarction in 27,000 participants from 52 countries: a case-control study. Lancet. 2005;366(9497):1640-9.

32. Silventoinen K, Jousilahti P, Vartiainen E, Tuomilehto J. Appropriateness of anthropometric obesity indicators in assessment of coronary heart disease risk among Finnish men and women. Scand J Public Health. 2003;31(4):283-90.

33. Mirmiran P, Esmaillzadeh A, Azizi F. Detection of cardiovascular risk factors by anthropometric measures in Tehranian adults: receiver operating characteristic (ROC) curve analysis. Eur J Clin Nutr. 2004;58(8):1110-8.

34. Stevens J. Ethnic-specific cutpoints for obesity vs countryspecific guidelines for action. Int J Obes Relat Metab Disord. 2003;27(3):287-8.

35. Razak F, Anand SS, Shannon H, Vuksan V, Davis B, Jacobs R, et al. Defining obesity cut points in a multiethnic population. Circulation. 2007;115(16):2111-8

36. Janghorbani M, Amini M, Willett WC, Mehdi Gouya M, Delavar A, Alikhani S, et al. First nationwide survey of prevalence of overweight, underweight, and abdominal obesity in Iranian adults. Obesity (Silver Spring). 2007;15(11):2797-808.

37. Heidari-Beni M, Hajimaghsood M, Ebrahimi-Mamaghani M, Jafarabadi MA, Mousavi-Jazayeri SM, Mohtadinia J. Diagnostic value of anthropometric indices for initial stage of atherosclerosis in adult women. Asia Pac J Clin Nutr. 2012;21(2):220-6.

38. Cikim AS, Ozbey N, Orhan Y. Relationship between cardiovascular risk indicators and types of obesity in overweight and obese women. J Int Med Res. 2004;32(3):268-73.

39. Yajnik CS, Yudkin JS. The YY paradox. Lancet. 2004;363(9403):163.

40. Lopez-Alvarenga JC, Montesinos-Cabrera RA, Velazquez-Alva C, Gonzalez-Barranco J. Short stature is related to high body fat composition despite body mass index in a Mexican population. Arch Med Res. 2003;34(2):137-40.

41. Lara-Esqueda A, Aguilar-Salinas CA, Velazquez-Monroy O, GomezPerez FJ, Rosas-Peralta M, Mehta R, et al. The body mass index is a less-sensitive tool for detecting cases with obesity-associated co-morbidities in short stature subjects. Int J Obes Relat Metab Disord. 2004;28(11):1443-50.

42. Fuchs FD, Gus M, Moreira LB, Moraes RS, Wiehe M, Pereira GM, et al. Anthropometric indices and the incidence of hypertension: a comparative analysis. Obes Res. 2005;13(9):1515-7.

43. Zhu S, Heshka S, Wang Z, Shen W, Allison DB, Ross R, et al. Com- 
bination of BMI and Waist Circumference for Identifying Cardiovascular Risk Factors in Whites. Obes Res. 2004;12(4):633-45.

44. Preis SR, Pencina MJ, D'Agostino RBS, Vasan RS, Levy D, Fox CS Neck circumference and development of cardiovascular disease risk factors in the Framingham heart study. Circulation. 2009;120(18 Supplement):S509.

45. Li HX, Zhang F, Zhao D, Xin Z, Guo SQ, Wang SM, et al. Neck circumference as a measure of neck fat and abdominal visceral fat in Chinese adults. BMC Public Health. 2014;14:311.

46. Aurora RN, Punjabi NM. Sleep Apnea and Metabolic Dysfunction: Cause or Co-Relation? Sleep Med Clin. 2007;2(2):237-50.
47. Roche F, Sforza E, Pichot V, Maudoux D, Garcin A, Celle S, et al. Obstructive sleep apnoea/hypopnea influences high-density lipoprotein cholesterol in the elderly. Sleep Med. 2009;10(8):882-6.

48. Santosa S, Jensen MD. Why are we shaped differently, and why does it matter? Am J Physiol Endocrinol Metab. 2008;295(3):E531-5.

49. Guo Z, Hensrud DD, Johnson CM, Jensen MD. Regional postprandial fatty acid metabolism in different obesity phenotypes. Diabetes. 1999;48(8):1586-92.

50. Zhou JY, Ge H, Zhu MF, Wang LJ, Chen L, Tan YZ, et al. Neck circumference as an independent predictive contributor to cardiometabolic syndrome. Cardiovasc Diabetol. 2013;12:76. 